[Agr. Biol. Chem., Vol. 34, No. 8, p. 1191 1197, 1970]

\title{
Isoenzymes of Japanese-radish Peroxidase ${ }^{\dagger}$
}

\author{
By Yuhei Morita, Chiaki Yoshida, Ikuo Kitamura and Shoji Ida \\ The Research Institute for Food Science, Kyoto University, Kyoto \\ Received January 23, 1970
}

\begin{abstract}
Japanese-radish root contained eighteen isoenzymes of peroxidase distinguishable on polyacrylamide gel electropherograms. The isoenzymes were found to be quite similar to those of horseradish peroxidase, although their quantities were different between two plants. The acidic components were the major isoenzyme in Japanese-radish peroxidase, while the neutral ones were the major one in horseradish. The chromatographic purification of the isoenzymes was performed on CM- and DEAE-Sephadex columns to characterize the components. The components in the preparations purified by the previously reported procedures of Morita et al. were also identified.
\end{abstract}

Morita and Kameda ${ }^{11}$ previously demonstrated that there are three isoenzymes in peroxidase of Japanese-radish (Raphanus sativus L.) root using paper electrophoretic technique, i.e. acidic, neutral and basic components. They have made a thorough investigation of chemical structure of the acidic component, which was designated as Japanese-radish peroxidase a $(J R P-a) .^{2 \sim 7)}$ They have also carried out a study on the basic component named as JRP-c. ${ }^{6 \sim 91}$ However, recent development in the systematic purification of enzyme proteins, e.g. ionexchange chromatography and gel electrophoresis, has prompted us the more thorough

$\dagger$ Studies on Phyto-peroxidase. Part XXIV.

1) X. Morita and K. Kameda, Mem. Research Inst. Food Sci, Kyoto Univ., 12, 1 (1957).

2) Y. Morita and K. Kameda, ibid., 12, 14 (1957); 14, 49, 61 (1958); 24, I (1962).

3) Y. Morita and K. Kameda, Bull. Agr. Chem. Soc. Japan, 23, 28 (1959).

4) Y. Morita, K. Shimizu and N. Kada, Agr. Biol. Chem., 32, 671 (1968).

5) Y. Morita, C. Yoshida and S. Ida, ibid., 33, 436 (1969).

6) K. Shimizu, T. Korenaga and Y. Morita, ibid., 29, 38 (1965).

7) Y. Morita, Y. Kominato and K. Shimizu, Mem. Research Inst. Food Sci., Kyoto Univ., 28, 1 (1967).

8) Y. Morita, K. Kameda and M. Mizuno, Agr. Biol. Chem., 25, 136 (1961); 26, 442 (1962).

9) K. Shimizu and Y. Morita, ibid., 30, 149 (1966). purification of each individual isoenzyme. Many reports have been presented on the isoenzymes of peroxidase of various plant tissues. Shannon et al. ${ }^{10 \sim 12)}$ isolated seven components of peroxidase isoenzymes from horseradish root (Armoracia rusticana) and compared the isoenzymes one another on their physicochemical and enzymatic properties. Both Japanese-radish and horseradish belong to the Cruciferae, and it is of great interest to compare the enzyme properties of peroxidase isoenzymes originated from both plants.

In this paper, we demonstrate the zymograms of JRP on polyacrylamide gel as well as those of horseradish peroxidase (HRP). The components which have been purified by the previously reported method of Morita et al. ${ }^{2,8)}$ are also identified.

\section{MATERIALS AND METHODS}

Materials. Japanese-radish roots harvested in Kyoto Prefecture in winter were purchased from the Central

10) L. M. Shannon, E. Kay and J. Y. Lew, J. Biol. Chem., 241, 2166 (1966).

11) E. Kay, L. M. Shannon and J. Y. Lew, ibid., 242, 2470 (1967).

12) E. H. Strickland, E. Kay, L. M. Shannon and J. Horwitz, ibid., 243, 3560 (1968). 
Market. About $100 \mathrm{~kg}$ roots were grated mechanically and squeezed out through cotton cloth. The juice was treated with ammonium sulfate (100\% saturation), and the precipitate was collected by filtration. The precipitate was dissolved in water and fractionated with ammonium sulfate. The precipitate at 30 to $100 \%$ saturation was collected and supplied for the systematic purification and identification of peroxidase isoenzymes by ion-exchange chromatography and polyacrylamide gel electrophoresis. The isolation and purification of the peroxidase isoenzymes in a large scale was performed separately from $4000 \mathrm{~kg}$ roots by a modification of the previous method.2,8)

Wild horseradish roots harvested in late autumn in Hokkaido area were supplied by Dr. Kiyoshi Kominato, to whom thanks are due. Ten $\mathrm{kg}$ roots were cut into small pieces and homogenized with 10 liters water. The homogenate was filtered through cotton cloth. The residue was again extracted with 15 liters $0.1 \mathrm{M}$

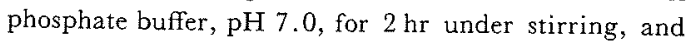
the slurry was filtered. The total extract was treated with ammonium sulfate similarly as in the case of preparing Japanese-radish peroxidase.

Polyacrylamide gel electrophoresis. Polyacrylamide gel electrophoresis was performed with a horizontal gel plate, $60 \times 90 \times 1 \mathrm{~mm}$, equilibrated with buffer solution. The gel plate containing $5.5 \%$ polyacrylamide was preliminarily soaked in a buffer solution overnight at room temperature in order to remove the unreacted materials in the gel. Each of Tris-citrate buffer, $\mathrm{pH} 8.7$, and borate buffer, $\mathrm{pH} \mathrm{8.2,} \mathrm{was} \mathrm{used} \mathrm{for} \mathrm{the}$ gel and the electrode vessels, respectively.13) In some cases $0.03 \mathrm{M}$ Tris-veronal buffer, $\mathrm{pH} 8.9$, or $0.025 \mathrm{M}$ acetate buffer, $\mathrm{pH} 4.8$, was also used for both gel and the electrode vessels. Electrophoresis was conducted for $2 \mathrm{hr}$ at $5^{\circ} \mathrm{C}$ at a potential gradient of 13 volts $\mathrm{cm}^{-1}$. Peroxidase samples were preliminarily deionized by dialysis and applied into the gels directly or by means of small pieces of cellulose acetate membrane dipped in the sample solution. At the termination of electrophoresis, peroxidase was located using benzidine and hydrogen peroxide reagent according to the method of Van Duijn.14)

Purification of peroxidase by ion-exchange chromatography. Ion-exchange chromatography on CM- and DEAESephadex columns was used for the purification of

13) M. D. Poulik, Nature, 180, 1477 (1957).

14) P. Van Duijn, Rec. Trav. Chim., 74, 771 (1955). peroxidase isoenzymes according to the method of Shannon et al.10 The peroxidase preparation was dialyzed against $0.005 \mathrm{M}$ acetate buffer, $\mathrm{pH} 4.4$, and put on a CM-Sephadex column, $2.5 \times 37 \mathrm{~cm}$, equilibrated with the same buffer. The acidic component of isoenzymes was passed through the column, while the neutral and basic isoenzymes were adsorbed on top of the column. The neutral isoenzymes were eluted by gradual increases of the buffer concentration and $\mathrm{pH}$ by using five compartments of an Autograd, Technicon Instrument Co. Chauncy, N.Y. The compartments contained $150 \mathrm{ml}$ each of acetate buffer, $\mathrm{pH} \mathrm{4.4,}$ of $0.005 \mathrm{M}, 0.05 \mathrm{M}, 0.1 \mathrm{M}$, and $0.2 \mathrm{M}$ concentrations, and $0.2 \mathrm{M}$ acetate buffer, $\mathrm{pH} 4.9$ (elution system 1). The basic isoenzymes remaining on the column were then eluted by the gradient of $\mathrm{pH}$ with two compartments containing $0.25 \mathrm{M}$ acetate buffer, $\mathrm{pH} 4.9$, and $0.2 \mathrm{M}$ phosphate buffer, $\mathrm{pH} 7.0$, respectively (elution system 2). Each of acidic, neutral, and basic fractions was again precipitated with ammonium sulfate and then dialyzed against buffer solutions for the further purification. The acidic fraction, Fraction A, was put on a DEAE-Sephadex column, $2.5 \times 38 \mathrm{~cm}$, equilibrated with $0.005 \mathrm{M}$ Tris- $\mathrm{HCl}$ buffer solution, pH 8.4. Peroxidase components were eluted by increasing the sodium chloride concentration in the buffer solution from zero to $0.1 \mathrm{M}$ linearly. The neutral components were chromatographed on a CM-Sephadex column with a lower gradient of buffer concentration. The column and the sample were equilibrated with $0.02 \mathrm{M}$ acetate buffer, $\mathrm{pH} 4.4$, and the gradient was formed between $0.02 \mathrm{M}$ and $0.1 \mathrm{M}$ acetate buffer, 500 $\mathrm{ml}$ each. The basic components were purified by CM-Sephadex chromatography according to the method reported previously ${ }^{81}$ Thus the column was first equilibrated with $\mathrm{M} / 30$ phosphate buffer, $\mathrm{pH} 6.0$, and the elution was performed by increasing buffer concentration and $\mathrm{pH}$.

Spectrophotometry. Spectrophotometric measurement was performed with a Beckman DU spectrophotometer and a Hitachi EPS-2 recording spectrophotometer.

Peroxidase activity. Two rate constants, $k_{1}$ for hydrogen peroxide and $k_{4}$ for guaiacol, were determined spectrophotometrically according to the method of Chance and Maehly ${ }^{15}$ at room temperature.

15) B. Chance and A. C. Maehly, "Methods in Enzymology," Vol. II, ed. by S. P. Colowick and N. O. Kaplan, Academic Press, 1955, p. 764. 


\section{RESULTS AND DISCUSSION}

Electrophoretic patterns of peroxidase isoenzymes of Japanese-radish root

Electropherograms of peroxidase isoenzymes of Japanese-radish root in three different buffer solutions are shown in Fig. 1a. The schematic patterns are also presented in Fig. 1b.
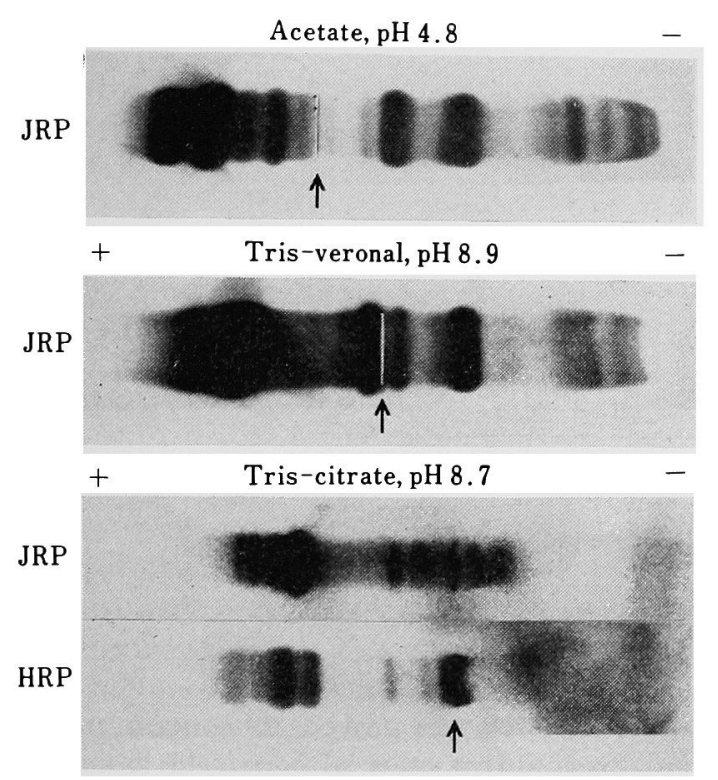

FIG. 1a. Electropherograms of Peroxidase Isoenzymes of Japanese-radish and Horseradish in Polyacrylamide Gels.

JRP and HRP represent the crude peroxidase preparations from Japanese-radish and horseradish roots, respectively (see the text). Arrows under each zymogram represent the origin. The buffer systems are described in the text.

Both figures show that there are many isoenzymes of peroxidase in this plant. On an electropherogram in acetate buffer, $\mathrm{pH} 4.8$, sixteen bands are distinguished, and two of them are further resolved into two components each by using either Tris-veronal or Tris-citrate buffer. Consequently, we conclude that there are eighteen isoenzymes. The band No. 3 is

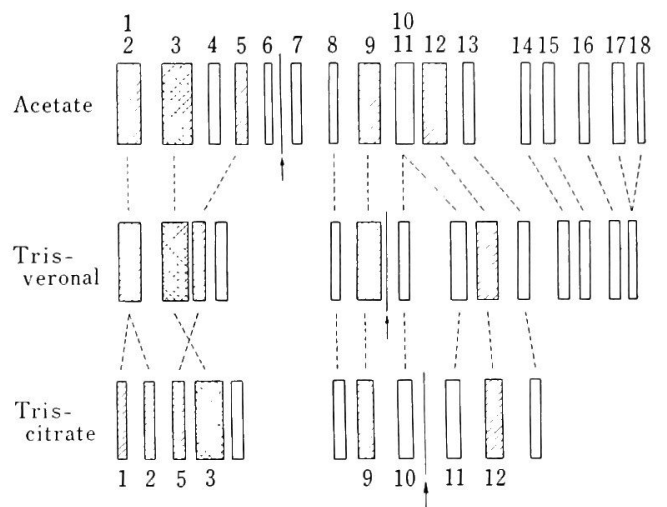

FIG. 1b. Schematic Electropherograms of Japaneseradish Peroxidase Isoenzymes in Polyacrylamide Gels with Various Buffers.

Arrows represent the origin. Shadowed components show strong activities on the electropherograms (see Fig. la and the text).

the major component of JRP of the crude extracts. The position of the component No. 5 replaces with that of the component No. 3 using Tris-citrate buffer solution ( $c f$. Fig. 1b). In an electropherogram using Tris-citrate buffer system, the basic components, No. 14 to 18 , were missing, because they are adsorbed to the cellulose acetate membrane remaining at the origin. By omitting the use of cellulose acetate membrane, however, these bands were observed, though they were much diffuse with tailing. Figure la shows also the zymogram of horseradish root. It can be seen that the position of some bands appears to correspond each other between two plants. There are nine isoenzymes of horseradish using Tris-citrate buffer, though the bands were less discrete compared with those of JRP. In this pattern as well the basic components were missing because of the adsorption at the origin. It is noted that relative intensities of the bands were different between two plant enzymes. In the case of HRP, a weakly basic or neutral isoenzymes were found to be the major component. 


\section{Purification of isoenzymes}

We first investigated the behaviors of isoenzymes during the systematic purification of the enzyme by ion-exchange chromatography. The systematic ion-exchange chromatography using CM- and DEAE-Sephadex according to Shannon et al. ${ }^{101}$ was found to be preferable for our experimental purpose. Figure 2 shows a typical chromatogram of the crude preparation on a CM-Sephadex column to separate into three fractions, $\mathrm{A}, \mathrm{B}$ and $\mathrm{C}$. The electropherograms of these three fractions are shown in Fig. 3. Fraction A passing through a CMSephadex column contained apparently the acidic isoenzymes, No. 1, 2, 3 and 5. Fraction $\mathrm{B}$ was eluted at higher concentrations of the buffer and was found to consist of one weakly acidic component, No. 5, and neutral components, No. 9, 11 and 12. Fraction C emerging at the buffer of higher concentra-

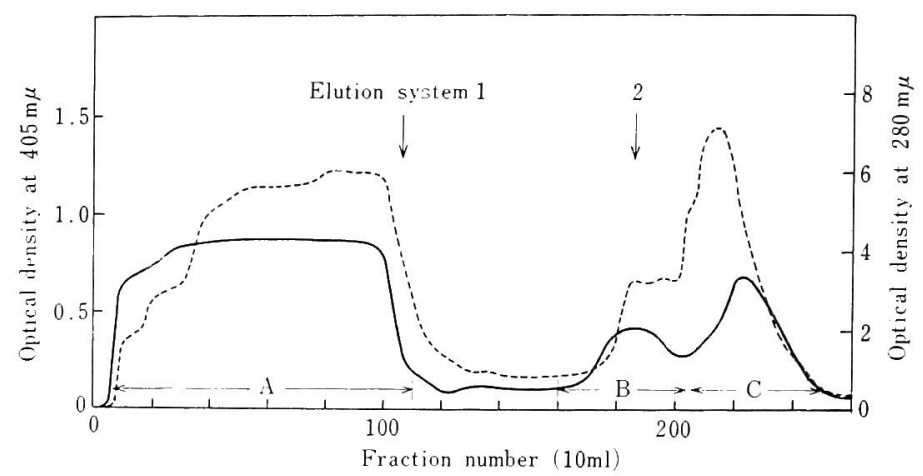

FIG. 2. Elution Profile of Crude Japanese-radish Peroxidase from a CM-Sephadex Column, $2.5 \times 37 \mathrm{~cm}$.

Elution systems 1 and 2: see text. Solid and dash lines represent the optical densities at 405 and $280 \mathrm{~m} \mu$, respectively.

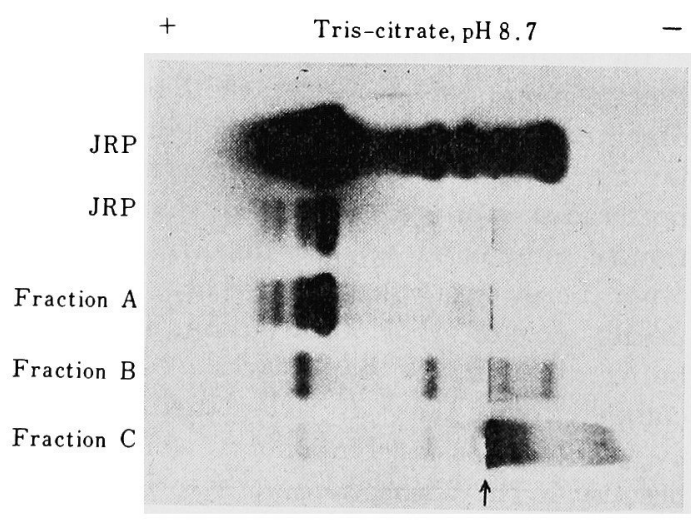

FIG. 3. Electropherograms of Peroxidase Fractions Isolated by CM-Sephadex Chromatography.

JRP denotes the crude peroxidase preparation from Japanese-radish. Fractions A, B, and C: see Fig. 2. Buffer system is Tris-citrate. tions and $\mathrm{pH}$ was proved to contain neutral and basic isoenzymes, although the basic components were not discrete because of tailing and adsorption at the origin.

Fraction A was next chromatographed on a column of DEAE-Sephadex, as shown in Fig. 4. By a gradual increase of the ionic strength of the buffer, three fractions were separated. The first fraction, A-1, was found to coincide with the major component, No. 3 , though it was little contaminated with the acidic isoenzyme. The second and third fractions, A-2 and A-3, were found to correspond with the more acidic isoenzymes, No. 2 and 1, respectively. However, the Fraction A-1 in Fig. 4 was not homogeneous but it was resolved into two components under lower gradient of ionic strength, as will be shown later 


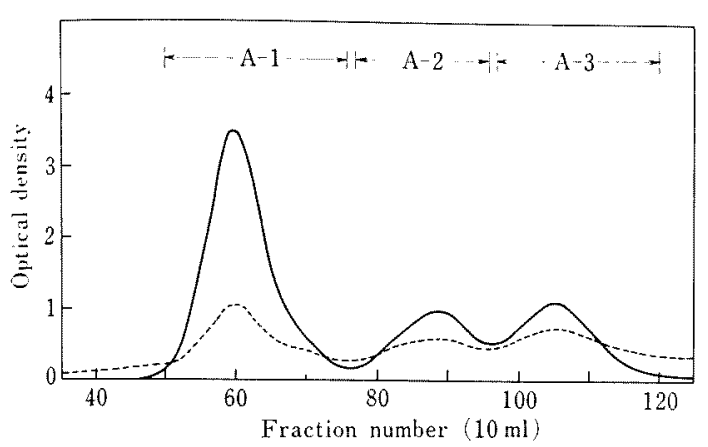

FIG. 4. Elution Profile of Acidic Fraction A from a DEAE-Sephadex Column, $2.5 \times 38 \mathrm{~cm}$.

Elution system: see the text. Solid and dash lines represent the optical densities at 405 and $280 \mathrm{~m} \mu$, respectively.

(Fig. 6). However, the further chromatographic purification and electrophoretic identification of Fractions B and $C$ was not possible. Then the purification of these neutral and basic components were undertaken using the previous method on a large scale.

Now, it appears to be pertinent to compare the previous purification method of peroxidase $^{2,81}$ with the chromatographic method presently employed. The classic method of preparing crystalline peroxidase a involved (1) fractionation with ammonium sulfate, (2) adsorption of impurities on tricalcium phosphate gels and alumina $C_{r}$ gels, (3) ethanol fractionation, (4) adsorption on alumina $C_{r}$ gels in the presence of $50 \%$ ethanol, and (5) crystallization with ammonium sulfate. In the present experiment, it was found that the method enabled us to concentrate predominantly the major acidic isoenzyme, No. 3. As yet the crystalline preparation obtained was not free from other isoenzymes. Figure 5 shows typical chromatograms of the crystalline preparation formed at from 0.53 to 0.60 saturation of ammonium sulfate and the fraction crystallized at less than 0.53 saturation. It is evident that the acidic isoenzymes passing through the column were contained in higher concentration in the preparation crystallized at more than
0.53 saturation of ammonium sulfate, while the neutral isoenzymes adsorbed on the column were concentrated in the fraction crystallized at less than 0.53 saturation. So the careful recrystallization will be partially effective to separate the neutral and acidic isoenzymes.

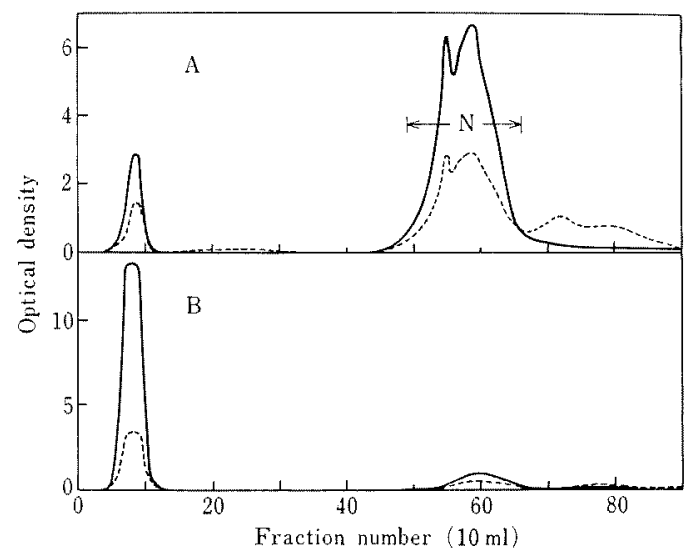

FIG. 5. Elution Profiles of Crystalline Peroxidase Preparations from a CM-Sephadex Column, $2.5 \times$ $36 \mathrm{~cm}$.

A: the preparation crystallized at 0.53 saturation of ammonium sulfate. B: the preparation crystallized at from 0.53 to 0.60 saturation. Elution system: the gradient was formed with four compartments containing $150 \mathrm{ml}$ each of $0.005 \mathrm{M}$, $0.1 \mathrm{M}, 0.1 \mathrm{M}$ and $0.25 \mathrm{M}$ acetate buffer, $\mathrm{pH} 4.4$. Solid and dash lines represent the optical densities at 405 and $280 \mathrm{~m} \mu$, respectively.

Among the acidic isoenzymes, No. 3 component was predominant in the crystalline preparation of peroxidase a, but the No. 3 component was resolved into two isoenzymes in the DEAE-Sephadex chromatography, as shown in Fig. 6. The first isoenzyme, A-la, was the major component, but the minor isoenzyme, $\mathrm{A}-1 \mathrm{~b}$, was indistinguishable in the electrophoretic analysis (Fig. 7). The more acidic components, No. 1 and 2, have been almost excluded in the crystalline preparation, but they were found to be concentrated in the supernatant fraction during the crystallization process. These components were also 


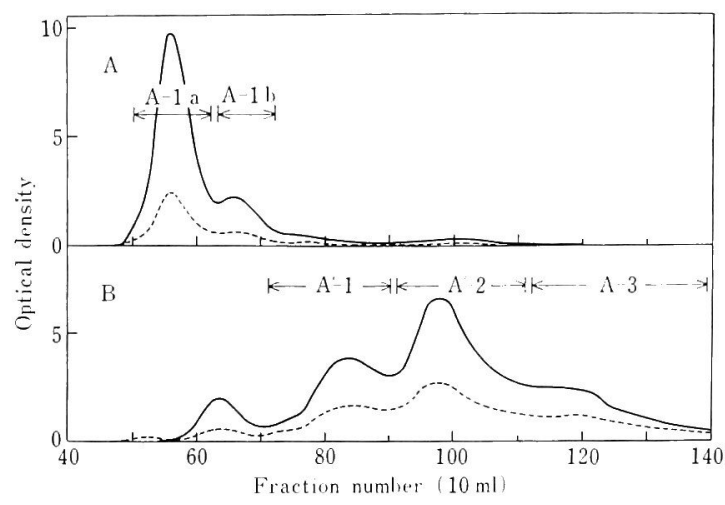

FIG. 6. Elution Profiles of Acidic Isoenzymes of Peroxidase from a DEAE-Sephadex Column, $2.5 \times$ $36 \mathrm{~cm}$.

A: acidic fraction from crystalline peroxidase (the first peak in Fig. 5B). B: acidic fraction from the similar CM-Sephadex chromatography on the supernatant fraction in the crystallization at 0.6 saturation. Elution system: the gradient was formed between $0.005 \mathrm{M}$ Tris buffer, $\mathrm{pH} 8.4$, and the same buffer containing $0.1 \mathrm{M}$ sodium chloride, 500 $\mathrm{ml}$ each, followed by further passing the latter solution. Solid and dash lines represent the optical densities at 405 and $280 \mathrm{~m} \mu$, respectively.

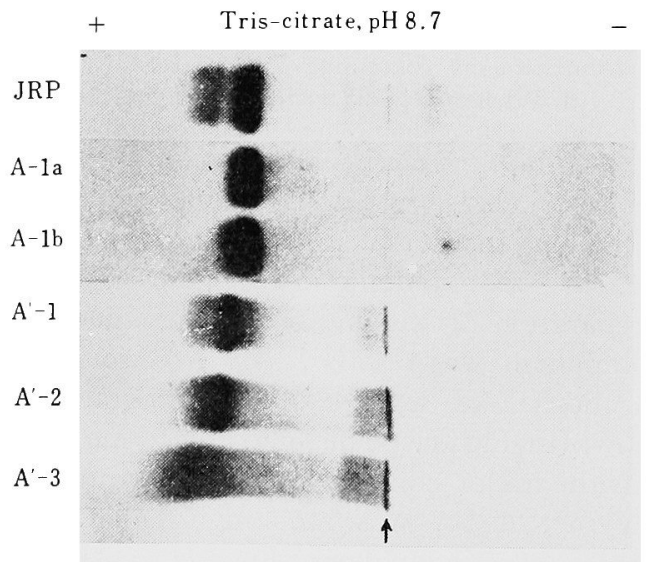

FIG. 7. Electropherograms of Purified Acidic Isoenzymes of Peroxidase.

A-1a, A-1b, $A^{\prime}-1, A^{\prime}-2$ and $A^{\prime}-3$ denote the fractions shown in Fig. 6. The buffer system is Tris-citrate. concentrated in the fraction which was not adsorbed on alumina $C_{r}$ gels in the presence of $50 \%$ ethanol. Figure 6 shows the chromatogram of this fraction. The purified components No. 1 and 2 did not exhibit discrete bands in electrophoresis because of large tailings, unlike the component No. 3 (Fig. 7).

Figure 8 shows a chromatogram of a neutral fraction, Fraction $\mathrm{N}$ in Fig. 5A, on a CMSephadex column. Two fractions were isolated as indicated by arrows in the figure. The first fraction $\mathrm{Nl}$, corresponded to the component No. 5 in the electrophoresis (Fig. 9). This fraction could be resolved into two or three components in the further chromatographic purification on a DEAE-Sephadex column, but the isolated components were not distinguished from one another on the electropherogram. The second fraction in Fig. 8,

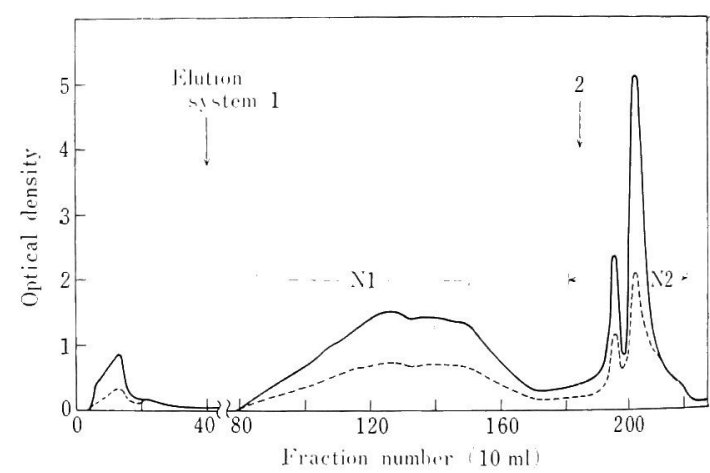

FIG. 8. Elution Profile of Neutral Isoenzymes from a CM-Sephadex Column, $2.5 \times 36 \mathrm{~cm}$.

Elution system 1: linear gradient formed between $500 \mathrm{ml}$ of $0.02 \mathrm{M}$ and $500 \mathrm{ml}$ of $0.08 \mathrm{M}$ acetate buffer, $\mathrm{pH}$ 4.4. Elution system $2: 0.08 \mathrm{M}$ acetate buffer, $\mathrm{pH}$ 4.4. Solid and dash lines represent the optical densities at 405 and $280 \mathrm{~m} \mu$, respectively.

N2, was rechromatographed on CM-Sephadex, and the corresponding fraction was obtained. This fraction contained several neutral components, No. 8, 9, 10, 11, 12 and 13, among which the No. 12 component was predominant. The further purification of these neutral com- 

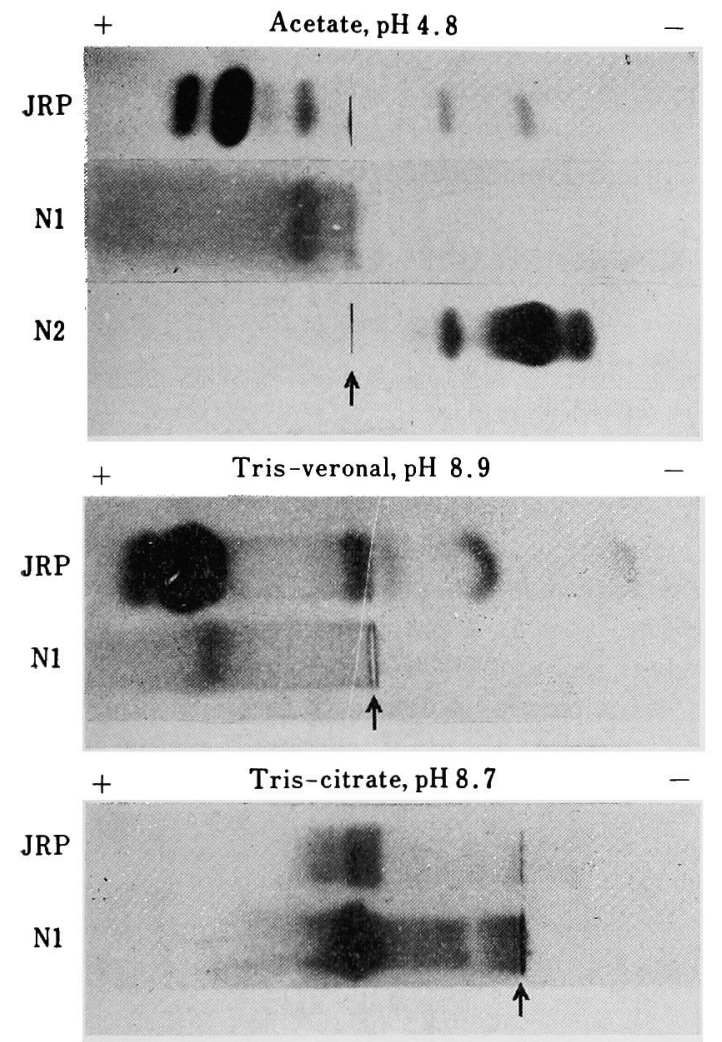

FIG. 9. Electropherograms of Purified Peroxidase Isoenzymes from Neutral Fraction.

$\mathrm{N} 1$ and $\mathrm{N} 2$ represent the fractions shown in Fig. 8. N 1 fraction was examined with three buffer systems.

ponents have not been performed.

The purification of the basic isoenzymes was performed according to the previous method ${ }^{81}$ using GM-Sephadex. Peroxidase c (emerging at tube no. 75 in Fig. 1 of literature 8) and the less basic isoenzyme (emerging at tube no. 60 in the same figure) were obtained both in crystalline form, and these isoenzymes

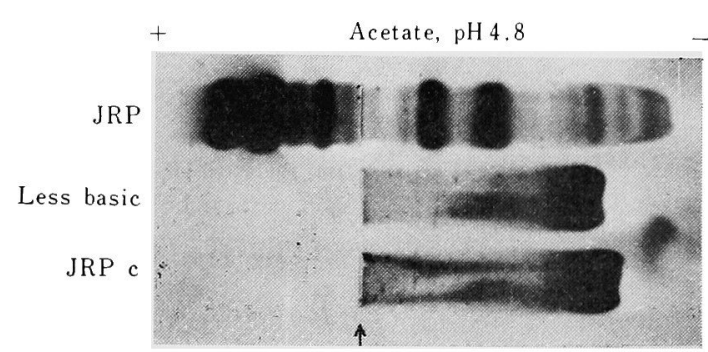

FIG. 10. Electropherograms of Basic Isoenzymes of Peroxidase.

JRP $\mathrm{c}$ and less basic component represent the crystalline peroxidase $c$ (No. 16 component) and the less basic component (No. 15 component), respectively (see the text). The buffer system is acetate.

were examined by electrophoresis. Figure 10 shows that these peroxidase isoenzymes correspond to the bands No. 16 and 15, respectively. Other minor basic isoenzymes have not been identified.

Finally, it should be noted that all of these isoenzymes occur in a single root. The physiological significance of the occurrence of many kinds of isoenzymes is unknown. However, we have found that the isoenzymes could be classified into the following three groups: acidic, neutral and basic ones, based upon their amino acid compositions as well as their catalytic properties and the behaviors of the heme group toward extrinsic ligands such as cyanide and hydroxyl ions. On the other hand, the microheterogeneity in each group seems to be derived from partly the differences in the number of amide groups and mostly the differences in the constitutions of carbohydrate moiety in the molecules. These comparative investigation on the properties and chemical compositions of the isolated isoenzymes will be presented in separate papers. 